\title{
Review on Therapeutic Efficacy of Mesenchymal Stem Cell (MSC) Therapy for Treating Different Disease
}

\author{
Farhana Hoque, Saima Akther, Md. Shahidul Islam* \\ Department of Pharmacy, University of Science and Technology Chittagong (USTC), Chattogram, Bangladesh.
}

How to cite this paper: Farhana Hoque, Saima Akther, Md. Shahidul Islam. (2021) Review on Therapeutic Efficacy of Mesenchymal Stem Cell (MSC) Therapy for Treating Different Disease. International Journal of Clinical and Experimental Medicine Research, 5(2), 96-100.

DOI: $10.26855 /$ ijcemr.2021.04.001

Received: December 25, 2020

Accepted: January 26, 2021

Published: February 23, 2021

*Corresponding author: Md. Shahidul Islam, Department of Pharmacy, University of Science and Technology Chittagong (USTC), Chattogram, Bangladesh.

Email: s_i_liton@yahoo.com

\begin{abstract}
Mesenchymal stem cells (MSCs) have been demonstrated as an attractive cell source for tissue engineering applications because of their ability to be easily isolated and expanded from adult bone marrow, umbilical cord, adipose tissue etc and their versatility for differentiation into multiple cells and tissues. The research is conducted by studying 80 different articles and journals focusing on the efficacy and success of MSC therapy for different disease like Vertebral Disc Regeneration, Craniofacial defects repairmen, Skeletal Muscle Repairmen, Cardiovascular disease, Osteoarthritis, Renal Disease, Type 1 Diabetes, Multiple sclerosis, liver disease, Tendon \& Ligament repair, Bone fracture and also for treating COVID patients. Reports of multiple clinical trials conducted upon more than 1,000 patients for treating diversified diseases has been included in this review to study the effectiveness and limitations of MSC Therapy. The results and discussion concluded with appreciative therapeutic outcome of most of the clinical trials conducted and there were few cases in which MSC Therapy failed to generate therapeutic response. Three major limitations and governing factor were found out to the reasons behind the success and failure of the therapy. This special issue has provided unprecedented insights into the roles of human MSC in treating numerous diseases and highlighted the remaining challenges and possible strategies to enhance their therapeutic potential. Despite significant advancements in MSC therapy understanding of the nature, function, mechanism, mode of isolation, and route of administration as well as experimental handling of MSCs is important to improve the therapeutic efficacy of MSCs. The therapeutic potential of MSCs will attract further research investments in this area to resolve the challenges and improve the effectiveness of medical treatment. Thus, it can be concluded that MSC Therapy can open a vast field of exploring regenerative drug.
\end{abstract}

\section{Keywords}

Mesenchymal, Review, Efficacy, Pharmacological Action

\section{Introduction}

Mesenchymal Stem cell (MSC) is also called mesenchymal stromal cells, are a subset of non-hematopoietic adult stem cells that originate from the mesoderm. These cells were first characterized by Friedenstein and his colleagues in 1974 [1]. MSCs are ubiquitously found throughout the human body and can be derived from multiple organ sys- 
tems including, but not limited to, bone marrow, heart, peripheral blood, adipose tissue, placenta, and umbilical cord [2]. Mesenchymal Stem cell therapy is an application of Tissue Engineering. First named in the 1980s by Arnold Caplan, mesenchymal stem cells (MSCs) and MSC based therapy have emerged as an extremely promising therapy in adult medicine and combined with a wealth of additional preclinical data [3]. Initially the attention towards MSC therapy began from the possibility of tissue regeneration and organ engineering based on the ability of MSCs to differentiate into bone and cartilage. Although some osteogenic and chondrogenic disorders do appear to benefit directly from tissue regeneration, newer preclinical and clinical trials suggests that MSCs instead represent "medicinal signaling cells" that secrete immunomodulatory, antiapoptotic, anti-inflammatory, proangiogenic, promitogenic, and antibacterial factors [4]. With the advancement of preclinical studies, MSCs have been shown to be effective in the treatment of many diseases, including both immune diseases and non-immune diseases. Stem cells can be used to treat a variety of diseases and several recent studies in animal models demonstrate the potential of bioengineering strategies targeting adult and embryonic stem cells [5]. In order to obtain the desired cells for transplantation, stem cell bioengineering approaches entail the manipulation of environmental signals influencing cell survival, proliferation, self-renewal and differentiation. In that regard, multivariate analytical approaches have been used with success to optimize different stem cell culture processes [6]. The genetic or molecular enhancement of stem cells is also a powerful means to control their proliferation or differentiation or to correct genetic defects in recipients. Tissue engineering is an interdisciplinary field which applies the principles of engineering and the life sciences in the development of biological substitutes that restore, maintain, or improve tissue function [7]. Every year millions of patients suffer the loss or failure of an organ or tissue as a result of accidents or disease. Over 8 million surgical procedures are performed to treat these patients in the U.S each year and the overall cost of these problems to the U.S economy is estimated to exceed $\$ 400$ billion per year [8]. Tissue or organ transplantation is a generally accepted therapy to treat these patients. An exciting strategy to treat patients who need a new organ or tissue is the engineering of man-made organs or tissues. Tissues or organs can be potentially engineered with a number of strategies [9].

\section{Materials and Methods}

A systematic review was done to examine the effects of MSCs therapy on multiple patients for different indications. The following databases were searched in: PubMed, MEDLINE, Google Scholar. All the searches were limited to literatures published between 1997 and 2020. Information about study objectives, participants and type of study were searched systematically with extensive keywords to optimize the sensitivity of data collection from 1974- 2020.

\section{Inclusion and Exclusion Criteria:}

Studies were included if they met all of the following criteria:

(1) Use of MSCs in at least one experimental group (animal models or clinical trials)

(2) Available in English language and

(3) Original data or a review

The flow of information from identification to inclusion of studies is summarized in following ways:

\section{Results and Discussion}

Donor variation in MSCs is unavoidable but variability can perhaps be minimised by determining standard selection criteria and MSC expansion methodology. Such measures are extremely necessary in order to ensure reproducibility of MSC preparations and a high level of therapeutic efficacy [10]. Wound healing is the physiologic response to a disruption in normal skin architecture and requires both spatial and temporal coordination of multiple cell types and cytokines. This complex process is prone to dysregulation secondary to local and systemic factors such as ischemia and diabetes that frequently lead to chronic wounds. Chronic wounds such as diabetic foot ulcers are epidemic with great cost to the healthcare system as they heal poorly and recur frequently, creating an urgent need for new and advanced therapies. Stem cell therapy is emerging as a potential treatment for chronic wounds [11]. A basic study using an influenza model and a clinical study on influenza A and COVID-19 showed promising results. With regard to the basic study on an influenza model it was reported the therapeutic efficacy of human umbilical cord tissue-derived MSCs using a model of acute lung injury (ALI) induced by the influenza A (H5N1) virus (12). Despite the success of this therapeutics, most of the MSC therapies either have had no success in late-stage clinical trials or did not progress beyond preclinical studies. While MSCs demonstrate an exceptional safety profile, they have generally been therapeutically ineffective in humans. It was confirmed that UC-MSCs were effective in restoring impaired alveolar fluid clearance and protein permeability of influenza A-infected alveolar epithelial cells. 
It was also confirmed that conditioned UC-MSC medium and UC-MSC exosomes have some therapeutic effects and only UC-MSCs slightly improved the survival of influenza A-infected mice [13]. A basic study using an influenza model and a clinical study on influenza A and COVID-19 showed promising results. With regard to the basic study on an influenza model it was reported the therapeutic efficacy of human umbilical cord tissue-derived MSCs (UC-MSCs) using a model of acute lung injury (ALI) induced by the influenza A (H5N1) virus. It was confirmed that UC-MSCs were effective in restoring impaired alveolar fluid clearance and protein permeability of influenza A-infected alveolar epithelial cells. It was also confirmed that conditioned UC-MSC medium and UC-MSC exosomes have some therapeutic effects and only UC-MSCs slightly improved the survival of influenza A-infected mice. With regard to the clinical study on severe COVID-19 cases, it was reported that seven cases (two common, four severe, and one critically severe case) were treated with MSCs. The trial concluded that MSCs significantly improved the functional outcome of all seven patients without any adverse effects. The pulmonary function of these seven patients significantly improved 2 days after MSC injection. Among them, two subjects with common COVID-19 and one subject with severe COVID-19 recovered and were discharged 10 days after treatment [14]. Although the number of COVID-19 patients who underwent MSC treatment is very limited and there is a lack of studies elucidating the underlying mechanisms, this study shows the potential application of MSC therapy for severe COVID-19 cases [15]. Several companies including Mesoblast, Athersys, Pluristem, Stempeutics, Cynata, and others are repurposing their MSC products for new indications. For example, Mesoblast has recently investigated the use of remestemcel-L to treat coronavirus disease 2019 (COVID-19) patients with moderate to severe ARDS, which is the major cause of death. The survival rate was $83 \%$ in ventilator-dependent COVID-19 patients when treated with two intravenous infusions of remestemcel-L. By comparison, the survival rate was only $12 \%$ in ventilator-dependent COVID-19 patients receiving standard of care during the same period [16].

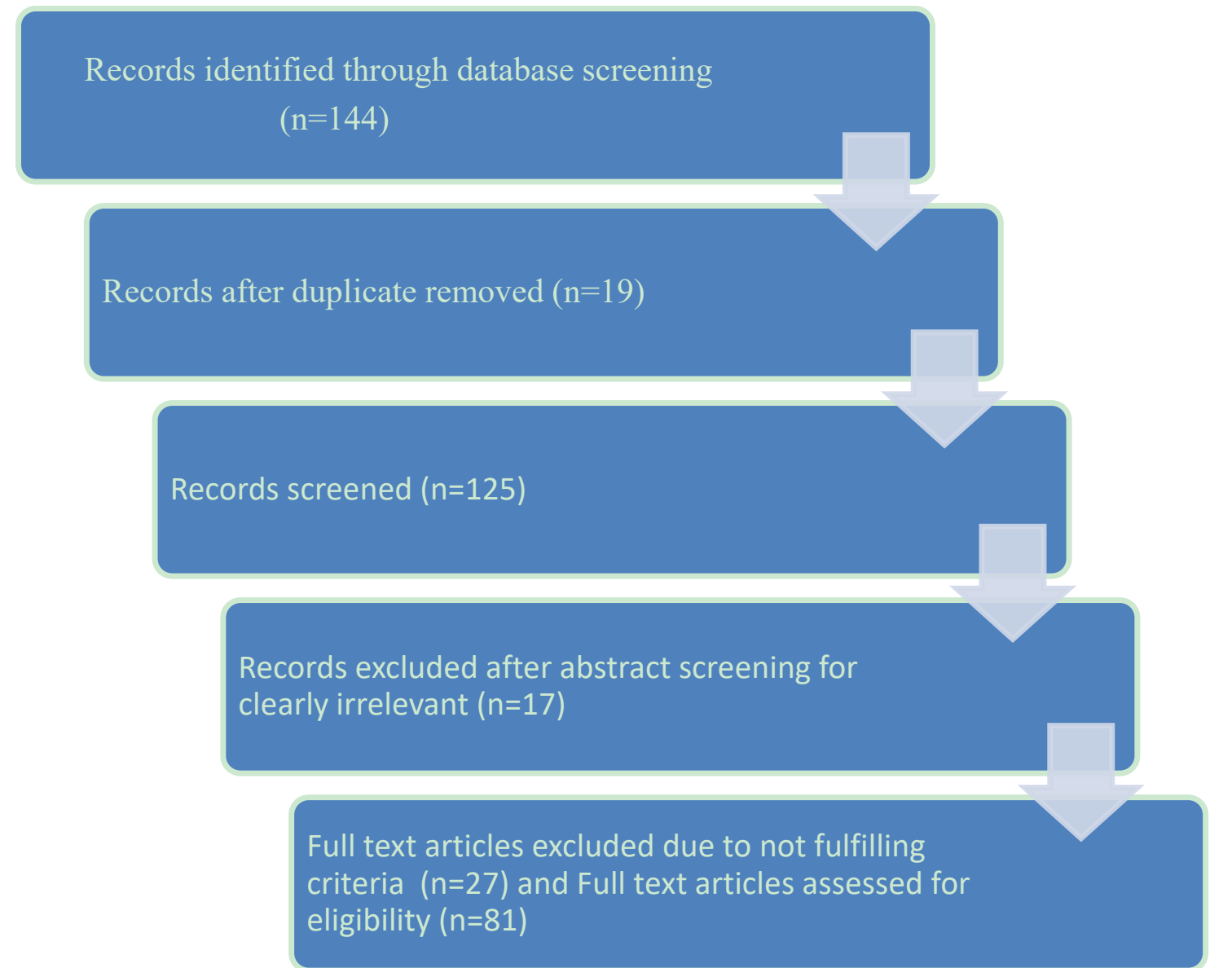

This review shows multiple successful clinical trials following MSC Therapy. The above given pie chart reflects the summary of diversified application of MSC Therapy for treating numerous diseases. From the Pie chart it is seen that the most application and most number of patients of MSC Therapy is in case of Bone related defects which is about $56 \%$. Here the bone related defects includes repair cranial defects, posterior spinal fusion $17 \%$ re- 
ferred to application and success of MSC Therapy in different kidney disease. Next $10 \%$ is comprised of the clinical trials conducted for Autoimmune disease which includes diseases like Rheumatoid Arthritis, Type 1 Diabetes, Psoriasis vulgaris and the total number of patients were 105. $8 \%$ was hold by Cardiovascular disease and Liver disease. A small percentage of application is observed in case of COVID patients [17]. However, in case of in vivo applications MSC still imposes limitations. There were some cases which were unsuccessful and did not provide any therapeutic response. 6 such cases were considered in the Findings part in which MSC did not fulfill the required expectations.

Some recent research demonstrated limited therapeutic effects of MSC treatment, suggesting that the direct regenerative potential of these cells related to their differentiation capacity may not be as effective as previously expected. Since several exogenous factors may greatly impact on the MSC biological properties and eventually on their therapeutic abilities, optimized protocols for MSC isolation and ex vivo preparation for clinical use need to be well established and standardized. Such comprehensive effort should be undertaken into consideration by a scientific community focusing on the practical MSC applications in tissue repair in terms of optimal preparation of MSC-based products for more effective therapies in patients [18].

This review also addresses the ways to overcome challenges in MSC Therapy. As the governing factors of the therapy depends on Donor. Administration process and Recipients therefore further research on these factors can lead to opening a wider field for MSC Therapy. From the future consumption reports it is clearly observed that the demand for MSC Pharmaceutical products will keep increasing in future.

\section{Conclusion}

Over the past decades, numerous studies have focused on developing therapeutic strategies based on cell therapy. As such a variety of tissue engineering approaches have been applied to develop advanced therapeutic strategies. This special issue known as Mesenchymal Stem cell has given unprecedented insights into the roles of human MSC in treating diversified diseases and highlighted the remaining challenges and possible strategies to enhance their therapeutic potential. Though MSC therapy has shown significant advancements, but some factors like deeper insights of the diverse origins of MSCs, the highly variable culture and cryopreservation conditions, the challenges associated with administration of MSCs, and the challenges of the host environment can also lead to unwanted therapeutic outcomes. Prolong exploration of engineering approaches that address these challenges should significantly improve the therapeutic efficacy for a broad range of clinical indications. Though there are many obstacles remain to overcome, I envision that the therapeutic potential of MSCs will attract further research investments in this area to overcome the challenges and facilitate the effectiveness of medical treatment. It can be anticipated that Mesenchymal stem cell therapy can confront many medical challenges facing humanity, increase our knowledge of pathogenesis, allow us to screen for new drugs for safety and effectiveness, and treat a variety of diseases. It is highly expected that deeper investigations of stem cell biology and clinical applicability will result in revolutions in medical technologies. Therefore, MSC Therapy can open a vast field for exploring regenerative medicine.

\section{References}

[1] Pountos, I., Corscadden, D., Emery, P., Giannoudis, P. V. (2007). Mesenchymal stem cell tissue engineering: techniques for isolation, expansion and application. Injury. 2007 Sep 1; 38: S23-33.

[2] Arthur, A., Zannettino, A., Gronthos, S. (2009). The therapeutic applications of multipotential mesenchymal/stromal stem cells in skeletal tissue repair. Journal of Cellular Physiology. 2009 Feb; 218(2): 237-45.

[3] Song, L., Baksh, D., Tuan, R. S. (2004). Mesenchymal stem cell-based cartilage tissue engineering: cells, scaffold and biology. Cyto therapy. 2004 Dec 1; 6(6): 596-601.

[4] Vacanti, J. P., Langer, R. (1999). Tissue engineering: the design and fabrication of living replacement devices for surgical reconstruction and transplantation. The lancet. 1999 Jul 1; 354: S32-4

[5] Audet, J. (2004). Stem cell bioengineering for regenerative medicine. Expert Opinion on Biological Therapy. 2004 May 1; 4(5): 631-44.

[6] Concise review: mesenchymal stem cell therapy for pediatric disease: perspectives on success and potential improvements. Stem Cells Translational Medicine. 2017 Feb; 6(2): 539-65.

[7] Lee, K. Y., Mooney, D. J. (2001). Hydrogels for tissue engineering. Chemical Reviews. 2001 Jul 11; 101(7): 1869-80.

[8] Steinert, A. F., Ghivizzani, S. C., Rethwilm, A., Tuan, R. S., Evans, C. H., Nöth, U. (2007). Major biological obstacles for persistent cell-based regeneration of articular cartilage. Arthritis Research \& Therapy. 2007 Jun 1; 9(3): 213.

[9] Jana, S., Levengood, S. K., Zhang, M. (2016). Anisotropic materials for skeletal-muscle-tissue engineering. Advanced Materi- 
als. 2016 Dec; 28(48): 10588-612.

[10] Mauney, J. R., Volloch, V., Kaplan, D. L. (2005). Role of adult mesenchymal stem cells in bone tissue engineering applications: current status and future prospects. Tissue Engineering. 2005 May 1; 11(5-6): 787-802.

[11] Saeedi, P., Halabian, R., Fooladi, A. A. (2019). A revealing review of mesenchymal stem cells therapy, clinical perspectives and modification strategies. Stem Cell Investigation. 2019; 6.

[12] McInnes, I. B., Schett, G. (2011). The pathogenesis of rheumatoid arthritis. New England Journal of Medicine. 2011 Dec 8; 365(23): 2205-19.

[13] Tsuchiya, A., Takeuchi, S., Iwasawa, T., Kumagai, M., Sato, T., Motegi, S., Ishii, Y., Koseki, Y., Tomiyoshi, K., Natsui, K., Takeda, N. (2020). Therapeutic potential of mesenchymal stem cells and their exosomes in severe novel coronavirus disease 2019 (COVID-19) cases. Inflammation and Regeneration. 2020 Dec; 40(1): 1-6.

[14] Loy, H., Kuok, D. I., Hui, K. P., Choi, M. H., Yuen, W., Nicholls, J. M., Peiris, J. M., Chan, M. C. (2019). Therapeutic Implications of Human Umbilical Cord Mesenchymal Stromal Cells in Attenuating Influenza A (H5N1) Virus-Associated Acute Lung Injury. The Journal of Infectious Diseases. 2019 Jan 7; 219(2): 186-96.

[15] Chen, J., Hu, C., Chen, L., Tang, L., Zhu, Y., Xu, X., Chen, L., Gao, H., Lu, X., Yu, L., Dai, X. (2020). Clinical study of mesenchymal stem cell treating acute respiratory distress syndrome induced by epidemic Influenza A (H7N9) infection, a hint for COVID-19 treatment. Engineering. $2020 \mathrm{Feb} 28$.

[16] Leng, Z., Zhu, R., Hou, W., Feng, Y., Yang, Y., Han, Q., Shan, G., Meng, F., Du, D., Wang, S., Fan, J. (2020). Transplantation of ACE2-mesenchymal stem cells improves the outcome of patients with COVID-19 pneumonia. Aging and Disease. 2020 Apr; 11(2): 216.

[17] Wei, A., Shen, B., Williams, L., Diwan, A. (2014). Mesenchymal stem cells: potential application in intervertebral disc regeneration. Translational Pediatrics. 2014 Apr; 3(2): 71.

[18] Lukomska, B., Stanaszek, L., Zuba-Surma, E., Legosz, P., Sarzynska, S., Drela, K. (2019). Challenges and controversies in human mesenchymal stem cell therapy. Stem Cells International. 2019 Apr 9. 